Research Paper

\title{
Fascin expression is inversely correlated with breast cancer metastasis suppressor 1 and predicts a worse survival outcome in node-negative breast cancer patients
}

\author{
Hye Jin Lee, Hee Jung An, Tae Hoen Kim, Gwangil Kim, Haeyoun Kang, Jin Hyung Heo, Ah-Young Kwon, \\ Sewha Kim $\bowtie$ \\ Department of Pathology, CHA Bundang Medical Center, CHA University, 59 Yatap-ro, Bundang-gu, Seongnam-si, Gyeonggi-do, 13496, Korea. \\ $\square$ Corresponding author: Sewha Kim MD, PhD; Address: Department of Pathology, CHA Bundang Medical Center, CHA University, 59 Yatap-ro, Bundang-gu, \\ Seongnam-si, Gyeonggi-do, 13496, Korea; Phone: +82-31-780-5499; Fax: +82-31-780-5476; Email: sewhakim@chamc.co.kr \\ (c) Ivyspring International Publisher. This is an open access article distributed under the terms of the Creative Commons Attribution (CC BY-NC) license \\ (https://creativecommons.org/licenses/by-nc/4.0/). See http://ivyspring.com/terms for full terms and conditions.
}

Received: 2017.07.23; Accepted: 2017.08.29; Published: 2017.09.06

\begin{abstract}
Background: Fascin is an actin-bundling protein that promotes cancer cell migration and invasion. By contrast, breast cancer metastasis suppressor 1 (BRMSI) inhibits cancer metastasis by targeting multiple steps of the metastatic cascade. We evaluated whether expression patterns of fascin and BRMSI correlate with clinicopathological features and patient outcome.

Methods: Immunohistochemistry for fascin and BRMSI was performed using a tissue microarray constructed from 183 human breast cancer tissues. Fascin expression determined by the proportion of stained tumor cells $(0: 0-5 \%, 1: 6-25 \%, 2: 26-50 \%, 3: 51-75 \%$, or $4:>75 \%)$ and staining intensity (0: negative, 1: weak, 2: moderate, or 3: strong) were multiplied and defined as negative (0-3) or positive (4-12). BRMS1 expression was scored separately based on nuclear and cytoplasmic staining intensity (0: negative, 1: weak, 2: moderate, 3: strong). We obtained the BRMSI H score by summing the nuclear and cytoplasmic scores and defined it as negative (0-2) or positive (3-6).

Results: Expression of BRMS1 showed a significant inverse correlation with that of fascin. Fascin ${ }^{+}$tumors were significantly associated with no lymph node metastasis, higher histological and higher nuclear grade, ER/PR/HER2 negativity, and triple-negative subtype (all ps $<0.05$ ). These clinicopathological differences

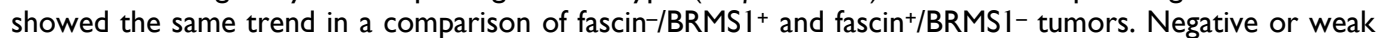
BRMS1 cytoplasmic expression was significantly associated with shorter disease-free survival (DFS; $p=0.043)$. Fascin positivity was significantly associated with shorter DFS $(p=0.005)$ and overall survival $(p=$ 0.020) when analyses were confined to node-negative patients.

Conclusions: This study confirms an inverse correlation between expression of fascin and expression of BRMS1 using a quite large cohort of human breast cancer tissues. Fascin alone or combined with BRMS1 was a worse prognostic marker, particularly in node-negative breast cancer patients.
\end{abstract}

Key words: breast cancer, fascin, breast cancer metastasis suppressor 1, immunohistochemistry

\section{Introduction}

The majority of cancer-related mortality (as much as $90 \%$ ) is associated with metastasis [1]. Acquisition of cell motility is the initial step in cancer invasion and metastasis, and aberrant regulation of the actin cytoskeleton in cancer cells is a fundamental mechanism enhancing cancer cell motility [2, 3]. Filopodia are actin-rich, slender projections on the cell surface that play an important role in cell migration. Fascin is an actin-bundling protein in filopodia that maximally cross-links actin filaments into tight bundles to provide filopodia with rigidity [4]. It is abundantly expressed in some types of normal cells, including neurons, glial cells, endothelial cells, and antigen-presenting dendritic cells [5]. Fascin 
overexpression is a poor prognostic indicator associated with aggressive clinicopathological features and metastasis in various types of human carcinomas, including breast cancers [6].

Breast cancer metastasis suppressor 1 (BRMS1) is a member of the family of metastasis suppressor genes initially discovered to reduce metastatic potential in a breast cancer xenograft model [7]. Subsequently it was found to suppress metastasis in other carcinomas, such as ovarian cancer, melanoma, rectal cancer, and non-small cell lung cancer. BRMS1 inhibits metastasis by targeting multiple steps of the metastatic cascade-cell adhesion, invasion, angiogenesis, and cytoskeletal rearrangementwithout affecting primary tumor growth [8-12].

A previous study demonstrated that fascin had an inhibitory effect on BRMS1 protein and an inverse correlation with the proteins in breast cancer [13]. However, no reported study has examined the relationship between expression patterns of these anti- and pro-metastatic molecules and patient outcome. We investigate expression patterns of fascin and BRMS1 in human breast cancer tissues and assess whether the two combinatorial markers reflect clinicopathological features.

\section{Materials and Methods}

\section{Patient selection and collection of clinicopathological data}

This study included 183 patients who underwent surgical treatment and were diagnosed with invasive breast cancer at CHA Bundang Medical Center from January 2006 to December 2008. Patients who underwent neoadjuvant chemotherapy before surgical treatment were excluded. Clinical data, including patient age at initial diagnosis, local recurrence, systemic recurrence, and patient survival, were retrieved from electronic medical records. This study was approved by the institutional review board of CHA Bundang Medical Center. Hematoxylin and eosin (H\&E)-stained slides of all cases were reviewed by two pathologists (HJ Lee and S Kim). Histological subtypes were classified based on the World Health Organization classification of breast tumors [14]. Histological grade was assessed using the Nottingham grading system [15]. Tumor stage was based on the 7th American Joint Committee on Cancer (AJCC) criteria [16].

\section{Tissue microarray (TMA) construction}

A representative H\&E-stained slide was selected for each case, and corresponding spots were marked on the surfaces of the paraffin blocks. Two core tissue biopsies ( $2 \mathrm{~mm}$ in diameter) were taken from each paraffin block and arranged in recipient TMA blocks using a trephine apparatus. A normal breast tissue core was included in each TMA block. Each tissue core was assigned a unique TMA number and linked to a database containing the clinicopathological data.

\section{Immunohistochemical staining and interpretation}

Immunohistochemistry (IHC) staining was performed on the TMA blocks using a mouse monoclonal antibody to fascin (Epitomics, Burlingame, CA, USA; 1:100) and rabbit polyclonal antibody to BRMS1 (Genetex, Irvine, CA, USA; 1:100). Briefly, $4 \mu \mathrm{m}$ TMA sections were transferred to adhesive slides and dried at $62^{\circ} \mathrm{C}$ for $30 \mathrm{~min}$. After incubation with primary antibodies, immunodetection was achieved with the addition of biotinylated anti-mouse and rabbit immunoglobulins, followed by peroxidase-labeled streptavidin (a component of a streptavidin-biotin kit), and 3,3'-diaminobenzidine as the chromogenic substrate. Slides were counterstained with Harris hematoxylin.

All IHC-stained slides were interpreted by two pathologists (HJ Lee and S Kim) under a multiview light microscope. Fascin IHC was scored with reference to the intensity and proportion of cytoplasmic staining of tumor cells as in previous studies [17-19]. The proportion of stained tumor cells (0: $0-5 \%, 1: 6-25 \%, 2: 26-50 \%, 3: 51-75 \%$, or $4:>75 \%)$ and staining intensity (0: no staining, 1 : weak, 2 : moderate, or 3: strong) were multiplied and defined as negative (0-3) or positive (4-12). Vascular endothelium exhibited strong staining for fascin and served as a positive internal control. Because BRMS1 expression pattern did not show intratumoral heterogeneity, only the staining intensity was considered in determining the BRMS1 expression level as in a previous study [20]. Nuclear and cytoplasmic staining intensities of BRMS1 were scored separately (0: no staining, 1: weak, 2: moderate, or 3: strong). We obtained the BRMS1 H score (range: 0-6) by summing the nuclear and cytoplasmic scores. Then we defined BRMS1 expression as negative (BRMS1 H score: 0-2) or positive (BRMS1 H score: 3-6). Normal breast epithelium exhibited strong staining for BRMS1 and served as a positive internal control for BRMS1 expression.

\section{Statistical analysis}

Data were analyzed using SPSS (ver. 20.0 for Windows; IBM Corp., Armonk, NY, USA). Fisher's exact test or the chi-square test was used to compare categorical variables. Student's $t$ test was used to compare continuous variables (BRMS1 $\mathrm{H}$ scores). A $p$-value of $<0.05$ indicated statistical significance. 
Survival analyses were performed using the Kaplan-Meier method with the log-rank test. Multivariate analyses were performed using Cox's regression model.

\section{Results}

\section{Baseline patient characteristics}

Clinicopathological characteristics of the patients and tumors are summarized in Table 1. Age at initial diagnosis was classified as $<50$ years $(62.3 \%)$ or $\geq 50$ years $(37.7 \%)$. Regarding tumor stage distribution, $48.6 \%$ and $51.4 \%$ of cases presented with pT1 and pT2-3. Lymph node metastasis (pN1-3) was observed in $45.4 \%$ of cases. Histological grade was 2 or 3 in $85.3 \%$ of cases. Nuclear grade was 2 or 3 in $60.1 \%$ of cases. The distribution of intrinsic subtypes was as follows: 101 luminal A (55.2\%), 18 luminal B (9.8\%), 22 HER2 (12.0\%), and 42 TNBC (23.0\%). Tumor recurrence, either local or systemic, occurred in 36 patients $(19.7 \%)$, and death from breast cancer occurred in 26 patients $(14.2 \%)$. The mean follow-up period was 87 months.

\section{Correlations between fascin expression, clinicopathological features, and BRMSI status}

Fascin positivity was observed in 33 of 183 patients $(18.0 \%)$. In analyses of the relationship between fascin status and various clinicopathological parameters (Table 1), fascin positivity was significantly associated with negative nodal metastasis $(p=0.003)$, higher histological grade $(p=$ $0.005)$, higher nuclear grade $(p=0.001)$, ER negativity $(p<0.001)$, PR negativity $(p<0.001)$, HER2 negativity $(p=0.018)$, and triple-negative subtype $(p<0.001)$. The inverse correlation between fascin and BRMS1 expression is shown in Figure 1. Although it was not statistically significant, BRMS1 nuclear expression tended to be negative or weak in fascin ${ }^{+}$tumors. Negative or weak BRMS1 cytoplasmic expression was observed more frequently in fascin ${ }^{+}$than in fascintumors $(p=0.012)$. A lower BRMS1 H score $(0-1)$ was observed more frequently in fascin ${ }^{+}$than in fascintumors $(p=0.031)$. The mean BRMS1 H score was also significantly lower in fascin ${ }^{+}(2.27 \pm 1.77)$ than in fascin- $^{-}(3.14 \pm 1.63)$ tumors $(p=0.008)$. Stratification of clinicopathological parameters by BRMS1 expression status revealed no statistically significant differences between the $\mathrm{BRMS1}^{+}$and BRMS1- groups (data not shown).

Table 1. Correlations between fascin expression and clinicopathological features

\begin{tabular}{|c|c|c|c|c|c|}
\hline \multirow[t]{2}{*}{ Parameter } & & \multirow[t]{2}{*}{ Number } & \multirow{2}{*}{$\begin{array}{l}\text { Fascin Negative } \\
\mathrm{n}=150\end{array}$} & \multirow{2}{*}{$\begin{array}{l}\text { Fascin Positive } \\
n=33\end{array}$} & \multirow[t]{2}{*}{$p$-value } \\
\hline & & & & & \\
\hline \multirow[t]{2}{*}{ Age } & $<50$ & $114(62.3)$ & $91(60.7)$ & $23(69.7)$ & 0.428 \\
\hline & $\geq 50$ & $69(37.7)$ & $59(39.3)$ & $10(30.3)$ & \\
\hline \multirow[t]{2}{*}{ Tumor stage } & pT1 & 89 (48.6) & $72(48.0)$ & $17(51.5)$ & 0.848 \\
\hline & pT2-3 & $94(51.4)$ & $78(52.0)$ & $16(48.5)$ & \\
\hline \multirow[t]{2}{*}{ Nodal stage } & pNo & $100(54.6)$ & $74(49.3)$ & $26(78.8)$ & 0.003 \\
\hline & pN1-3 & $83(45.4)$ & $76(50.7)$ & $7(21.2)$ & \\
\hline \multirow[t]{2}{*}{ AJCC stage } & I & 148 (80.9) & $118(79.4)$ & $30(90.9)$ & 0.142 \\
\hline & II-III & 35 (19.1) & $32(21.3)$ & $3(9.1)$ & \\
\hline \multirow[t]{2}{*}{ Histologic grade } & 1 & $26(14.2)$ & $26(17.3)$ & $0(0.0)$ & 0.005 \\
\hline & $2 / 3$ & $157(85.8)$ & $124(82.7)$ & $33(100.0)$ & \\
\hline \multirow[t]{2}{*}{ Nuclear grade } & 1 & $73(39.9)$ & $68(45.3)$ & $5(15.2)$ & 0.001 \\
\hline & $2 / 3$ & $110(60.1)$ & $82(54.7)$ & $28(84.8)$ & \\
\hline \multirow[t]{2}{*}{ Lymphovasculr invasion } & Absent & $79(43.2)$ & $60(40.0)$ & $19(57.6)$ & 0.081 \\
\hline & Present & $104(56.8)$ & $90(60.0)$ & $14(42.4)$ & \\
\hline \multirow[t]{2}{*}{ ER } & Positive & 117 (63.9) & $114(76.0)$ & $3(9.1)$ & $<0.001$ \\
\hline & Negative & $66(36.1)$ & $36(24.0)$ & $30(90.9)$ & \\
\hline \multirow[t]{2}{*}{ PR } & Positive & $94(51.4)$ & $91(60.7)$ & $3(9.1)$ & $<0.001$ \\
\hline & Negative & 89 (48.6) & $59(39.3)$ & $30(90.9)$ & \\
\hline \multirow[t]{2}{*}{ HER2 } & Negative & $143(78.1)$ & $112(74.7)$ & 31 (93.9) & 0.018 \\
\hline & Positive & $40(21.9)$ & 38 (25.3) & $2(6.1)$ & \\
\hline \multirow[t]{4}{*}{ Intrinsic subtype } & Luminal A & $101(55.2)$ & 98 (65.3) & $3(9.1)$ & $<0.001$ \\
\hline & Luminal B & $18(9.8)$ & $18(12.0)$ & $0(0.0)$ & \\
\hline & HER2 & $22(12.0)$ & $20(13.3)$ & $2(6.1)$ & \\
\hline & Triple-negative & $42(23.0)$ & $14(9.3)$ & $28(84.8)$ & \\
\hline Tumor recurrence & Yes & 36 (19.7) & $28(18.7)$ & $8(24.2)$ & 0.473 \\
\hline Patients death & Yes & $26(14.2)$ & $20(13.3)$ & $6(18.0)$ & 0.581 \\
\hline
\end{tabular}


A Number of pationts

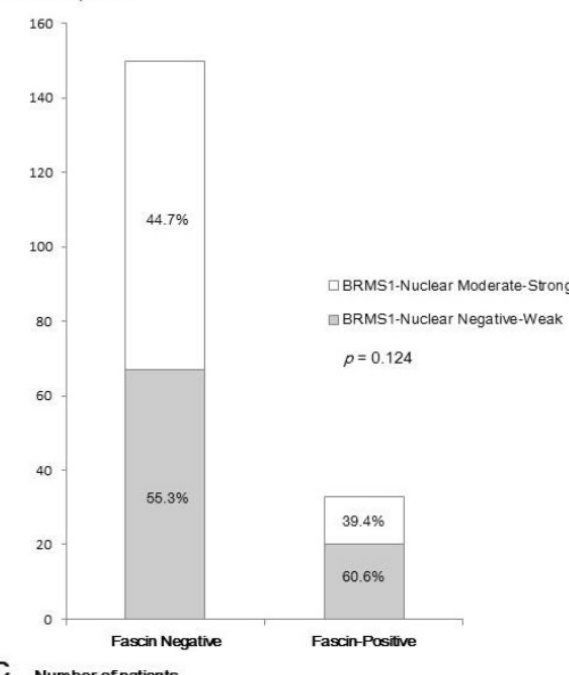

C Number of patients

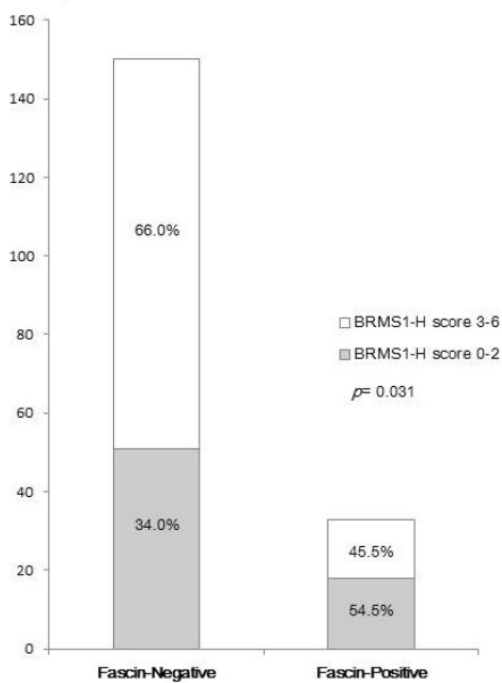

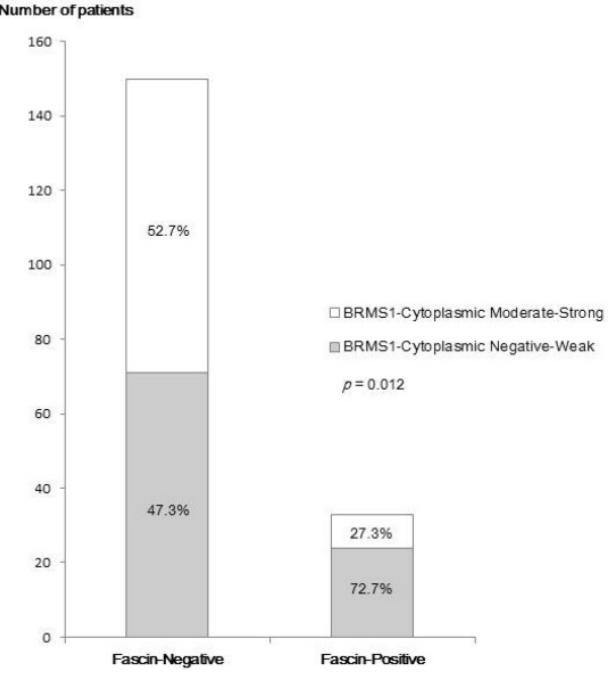

D

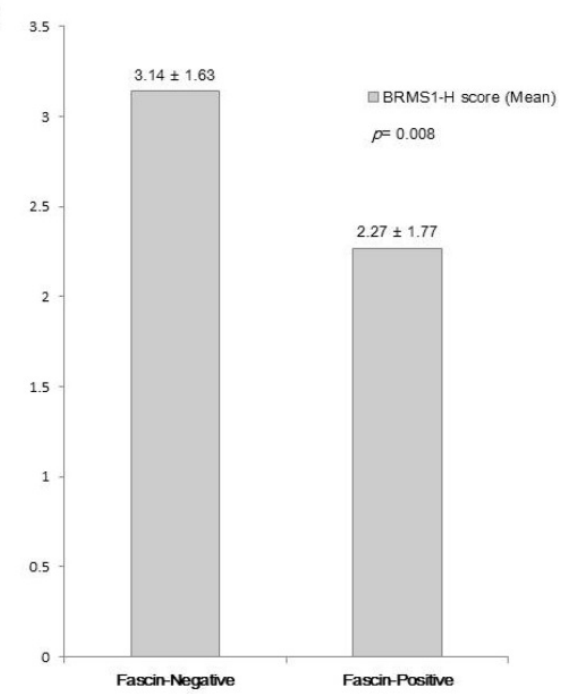

Figure 1. A comparison of the distribution of BRMS1 expression status between fascin- and fascin ${ }^{+}$breast cancers. (a) Distribution of nuclear BRMS1 expression, (b) distribution of cytoplasmic BRMSl expression, (c) distribution of low and high BRMS1 H scores, (d) difference in mean BRMS1 H scores.

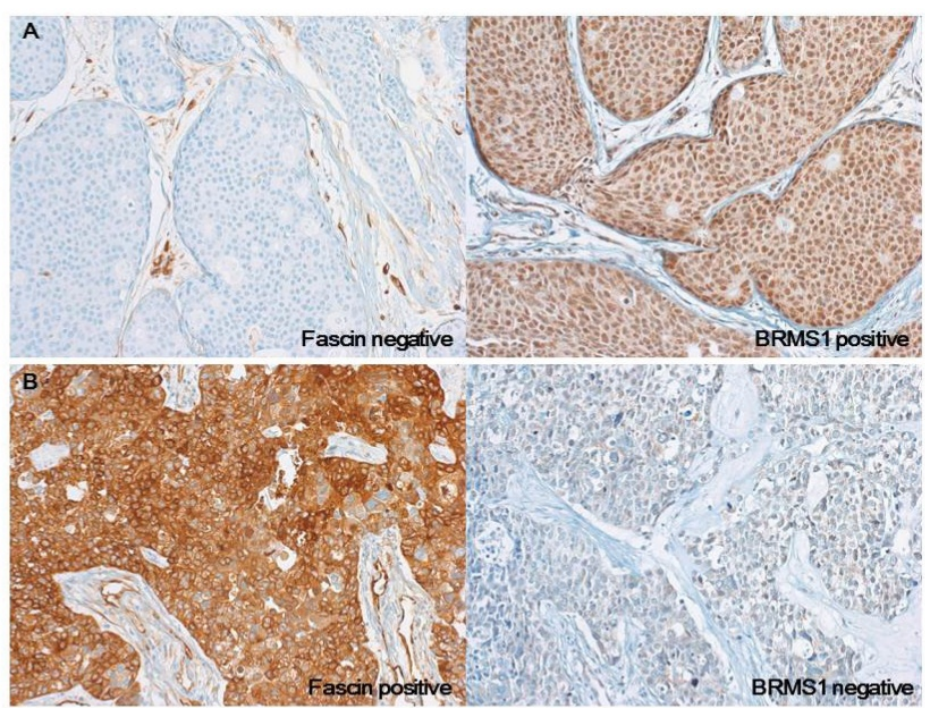

Figure 2. Photomicrographs of representative cases of fascin-/BRMS1+ and fascin+/BRMS1- breast cancers. (a) In contrast to stromal endothelial cells, which are normal internal controls, no fascin staining is observed in tumor cells. BRMS1 is stained in both nucleus and cytoplasm, but nuclear staining intensity is stronger than cytoplasm in this case. (b) Strong cytoplasmic fascin staining is observed in tumor cells, whereas BRMS1 is almost completely disappeared in the nucleus and is stained very faintly only in the cytoplasm 


\section{Clinicopathological differences according to fascin and BRMSI expression}

The distribution according to fascin and BRMS1 staining results was as follows: $51 \mathrm{fascin}^{-} / \mathrm{BRMS1}^{-}, 99$ fascin-/BRMS1 ${ }^{+}, 18$ fascin $^{+} /$BRMS1- $^{-}$, and 15 fascin ${ }^{+} / \mathrm{BRMS1}^{+}$. Compared to the fascin-/BRMS1 ${ }^{+}$ subgroup, the fascin ${ }^{+}$BRMS1- $^{-}$subgroup was significantly associated with negative nodal metastasis $(p=0.038)$, higher histological grade $(p=$ $0.040)$, higher nuclear grade $(p=0.008)$, ER negativity $(p<0.001)$, PR negativity $(p<0.001)$, and triple-negative subtype $(p<0.001)$ (Table 2). The representative cases of fascin ${ }^{-} / \mathrm{BRMS1}^{+}$and fascin $^{+}$/ BRMS1- tumors are depicted in Figure 2.

\section{Survival outcome according to clinicopathological differences and fascin/BRMS1 status}

Univariate analyses of all patients $(n=183)$ revealed that factors associated with shorter disease-free survival (DFS) were nodal metastasis $(p=$ 0.005), higher AJCC stage $(p=0.002)$, higher histological grade $(p=0.006)$, and negative or weak BRMS1 cytoplasmic expression $(p=0.043)$. Factors associated with shorter overall survival (OS) were higher T stage $(p=0.003)$, nodal metastasis $(p=0.004)$, higher AJCC stage $(p<0.001)$, and higher histological grade $(p=0.027)$. Then we performed multivariate Cox regression analyses on the prognostic factors identified in the univariate analyses. In multivariate analyses, nodal metastasis (hazard ratio $[\mathrm{HR}]=1.811$; 95\% confidence interval $[\mathrm{CI}]=0.833-4.201 .56$; $p=0.020)$ and higher AJCC stage ( $\mathrm{HR}=2.854 ; 95 \% \mathrm{CI}$ $=1.212-4.812 ; p=0.025)$ significantly increased the likelihood of tumor recurrence, whereas higher AJCC stage $(\mathrm{HR}=3.159 ; 95 \% \mathrm{CI}=1.460-6.834 ; p=0.003)$ was the only factor that significantly increased the likelihood of patient death (Table 3).

Survival analyses were also performed, confined to the patients with nodal metastasis $(n=117$, Table 3 and Figure 3). Fascin positivity was associated with shorter DFS in both univariate $(p=0.005)$ and multivariate $(\mathrm{HR}=5.583 ; 95 \% \mathrm{CI}=1.318-23.655 ; p=$ 0.020 ) analyses. Factors associated with shorter OS were higher T stage $(p=0.048)$, TNBC subtype $(p=$ $0.028)$, fascin positivity $(p=0.031)$, and fascin $^{+}$/BRMS1- subgroup ( $\left.p=0.041\right)$. In multivariate analyses, higher $\mathrm{T}$ stage $(\mathrm{HR}=5.622 ; 95 \% \mathrm{CI}=$ 1.055-29.968; $p=0.043$ ) and fascin positivity $(\mathrm{HR}=5.869 ; 95 \% \quad \mathrm{CI}=1.202-28.662 ; p=0.029)$ significantly increased the likelihood of patient death.
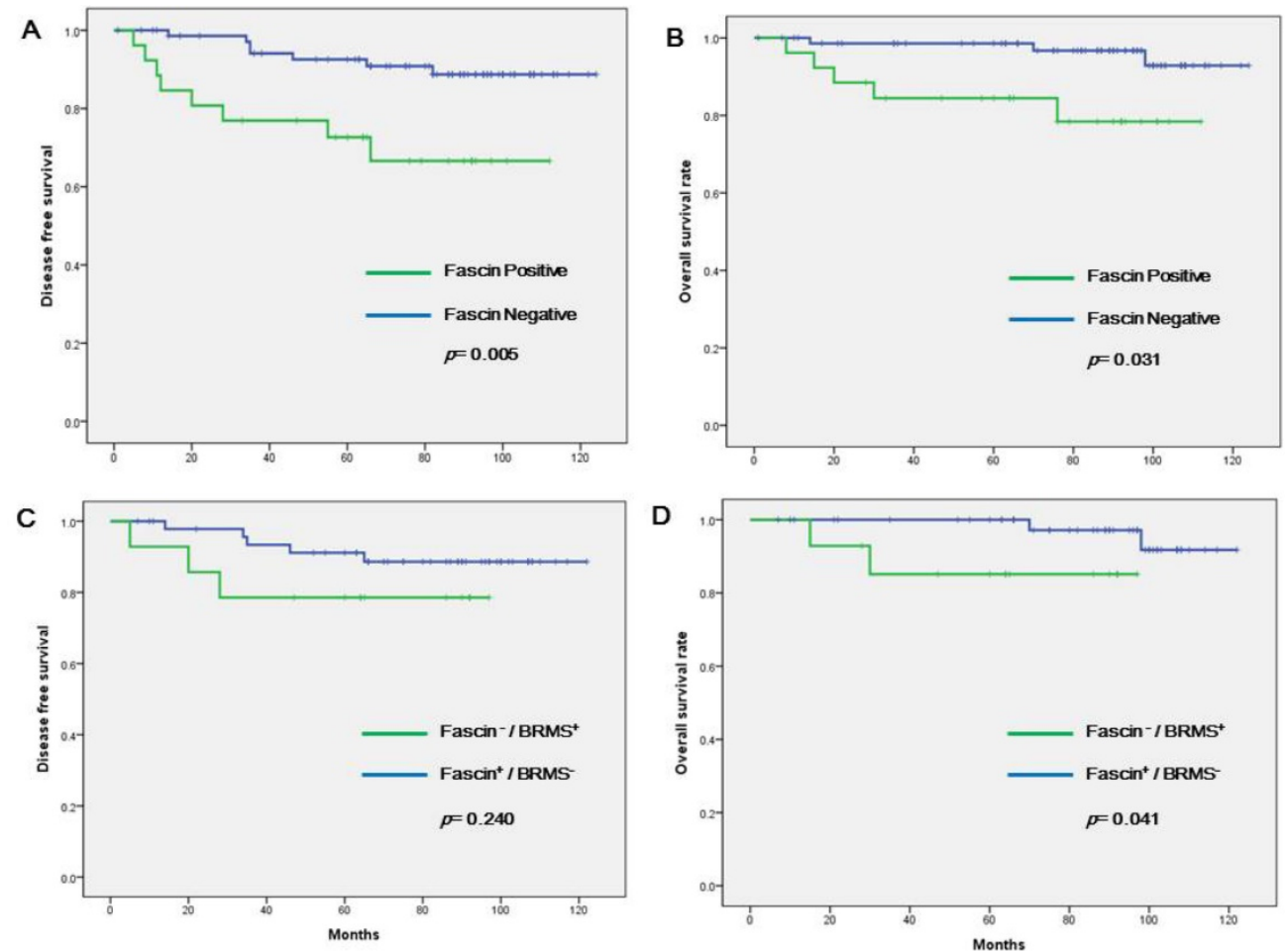

Figure 3. Kaplan-Meier survival curves analyzed in node-negative patients only. (a) Disease-free survival (DFS) according to fascin status, (b) overall survival (OS) according to fascin status, (c) difference in DFS between fascin $-/ B R M S 1^{+}$and fascin ${ }^{+} / B R M S 1^{-}$patients, (d) difference in OS between fascin $/$BRMS1 ${ }^{+}$and fascin+/BRMS1- patients. 
Table 2. Correlations between combined fascin and BRMS1 expression status and clinicopathological features

\begin{tabular}{|c|c|c|c|c|}
\hline \multirow[t]{2}{*}{ Parameter } & & \multirow{2}{*}{$\begin{array}{l}\text { Fascin-/BRMS1+ } \\
\mathrm{n}=99\end{array}$} & \multirow{2}{*}{$\begin{array}{l}\text { Fascin+/BRMS1- } \\
\mathrm{n}=18\end{array}$} & \multirow[t]{2}{*}{$p$-value } \\
\hline & & & & \\
\hline \multirow[t]{2}{*}{ Age } & $<50$ & $61(61.6)$ & $13(72.2)$ & 0.439 \\
\hline & $\geq 50$ & $38(38.4)$ & $5(27.8)$ & \\
\hline \multirow[t]{2}{*}{ Tumor stage } & pT1 & 49 (49.5) & $8(44.4)$ & 0.800 \\
\hline & pT2-3 & $50(50.5)$ & $10(55.6)$ & \\
\hline \multirow[t]{2}{*}{ Nodal stage } & pNO & 49 (49.5) & $14(77.8)$ & 0.038 \\
\hline & pN1-3 & $50(50.5)$ & $4(22.2)$ & \\
\hline \multirow[t]{2}{*}{ AJCC stage } & I & $79(79.8)$ & $15(83.3)$ & 1.000 \\
\hline & II-III & $20(20.2)$ & $3(16.7)$ & \\
\hline \multirow[t]{2}{*}{ Histologic grade } & 1 & $21(21.2)$ & $0(0.0)$ & 0.040 \\
\hline & $2 / 3$ & $78(78.8)$ & $18(100.0)$ & \\
\hline \multirow[t]{2}{*}{ Nuclear grade } & 1 & $44(44.4)$ & $2(11.1)$ & 0.008 \\
\hline & $2 / 3$ & $55(55.6)$ & $16(88.9)$ & \\
\hline \multirow[t]{2}{*}{ Lymphovasculr invasion } & Absent & $40(40.4)$ & $10(55.6)$ & 0.302 \\
\hline & Present & $59(59.6)$ & $8(44.4)$ & \\
\hline \multirow[t]{2}{*}{ ER } & Positive & $75(75.8)$ & $0(0.0)$ & $<0.001$ \\
\hline & Negative & $24(24.2)$ & $18(100.0)$ & \\
\hline \multirow[t]{2}{*}{ PR } & Positive & $59(59.6)$ & $0(0.0)$ & $<0.001$ \\
\hline & Negative & $40(40.4)$ & $18(100.0)$ & \\
\hline \multirow[t]{2}{*}{ HER2 } & Negative & $73(73.7)$ & $17(94.4)$ & 0.069 \\
\hline & Positive & $26(26.3)$ & $1(5.6)$ & \\
\hline \multirow[t]{4}{*}{ Intrinsic subtype } & Luminal A & $63(63.6)$ & $0(0.0)$ & $<0.001$ \\
\hline & Luminal B & $13(13.1)$ & $0(0.0)$ & \\
\hline & HER2 & $13(13.1)$ & $1(5.6)$ & \\
\hline & Triple-negative & $10(10.1)$ & $17(94.4)$ & \\
\hline Tumor recurrence & Present & $83(83.8)$ & $16(88.9)$ & 0.738 \\
\hline Patients death & Present & $12(12.1)$ & $3(16.7)$ & 0.700 \\
\hline
\end{tabular}

Table 3. Univariate and multivariate analyses of disease-free and overall survival

\begin{tabular}{|c|c|c|c|c|c|c|}
\hline \multirow[t]{3}{*}{ Parameters } & \multicolumn{3}{|c|}{ Disease free survival } & \multicolumn{3}{|c|}{ Overall survival } \\
\hline & \multirow{2}{*}{$\frac{\text { Univariate }}{p \text {-value }}$} & \multicolumn{2}{|c|}{ Multivariate } & \multirow{2}{*}{$\begin{array}{l}\text { Univariate } \\
p \text {-value }\end{array}$} & \multicolumn{2}{|c|}{ Multivariate } \\
\hline & & $\mathrm{HR}(95 \% \mathrm{CI})$ & $p$-value & & HR $(95 \% \mathrm{CI})$ & $p$-value \\
\hline \multicolumn{7}{|l|}{ Analysis in all patients $(n=183)$} \\
\hline Age $(<50$ vs. $\geq 50)$ & 0.802 & & & 0.249 & & \\
\hline T stage (pT1 vs. pT2-3) & 0.098 & & & 0.003 & $2.047(0.759-5.520)$ & 0.157 \\
\hline N stage (pN0 vs. pN1-3) & 0.005 & $1.811(0.833-4.201)$ & 0.020 & 0.004 & $1.613(0.563-4.619)$ & 0.373 \\
\hline AJCC stage (I vs. II-III) & 0.002 & $2.854(1.212-4.812)$ & 0.025 & $<0.001$ & $3.159(1.460-6.834)$ & 0.003 \\
\hline Histologic grade (1 vs. $2-3$ ) & 0.006 & $1.332(0.926-3.278)$ & 0.186 & 0.027 & $1.533(0.823-3.554)$ & 0.974 \\
\hline Nuclear grade (1 vs. $2-3$ ) & 0.055 & & & 0.103 & & \\
\hline Intrinsic subtype (non-TNBC vs. TNBC) & 0.074 & & & 0.589 & & \\
\hline Fascin (Negative vs. Positive) & 0.317 & & & 0.345 & & \\
\hline BRMS1-nuc (Moderate/Strong vs. Negative/Weak) & 0.988 & & & 0.415 & & \\
\hline BRMS1-cyt (Moderate/Strong vs. Negative/Weak) & 0.043 & $1.875(0.949-3.702)$ & 0.070 & 0.145 & & \\
\hline BRMS1-H score (3-6 vs. 0-2) & 0.769 & & & 0.492 & & \\
\hline Fascin-/BRMS1+ vs. Fascin+/BRMS1- & 0.698 & & & 0.430 & & \\
\hline \multicolumn{7}{|l|}{ Analysis in node-negative patients $(n=117)$} \\
\hline Age $(<50$ vs. $\geq 50)$ & 0.463 & & & 0.335 & & \\
\hline T stage (pT1 vs. pT2-3) & 0.258 & & & 0.048 & $5.622(1.055-29.968)$ & 0.043 \\
\hline Histologic grade (1 vs. $2-3$ ) & 0.050 & & & 0.155 & & \\
\hline Nuclear grade (1 vs. $2-3)$ & 0.136 & & & 0.549 & & \\
\hline Intrinsic subtype (non-TNBC vs. TNBC) & 0.125 & & & 0.028 & $2.030(0.127-32.417)$ & 0.617 \\
\hline Fascin (Negative vs. Positive) & 0.005 & $5.583(1.318-23.655)$ & 0.020 & 0.031 & $5.869(1.202-28.662)$ & 0.029 \\
\hline BRMS1-nuc (Moderate/Strong vs. Negative/Weak) & 0.121 & & & 0.145 & & \\
\hline BRMS1-cyt (Moderate/Strong vs. Negative/Weak) & 0.470 & & & 0.619 & & \\
\hline BRMS1-H score (3-6 vs. 0-2) & 0.321 & & & 0.717 & & \\
\hline Fascin-/BRMS1+ vs. Fascin+/BRMS1- & 0.240 & & & 0.041 & $1.185(0.151-9.294)$ & 0.871 \\
\hline
\end{tabular}

nuc nuclear, cyt cytoplasmic, $H R$ hazard ratio, $C I$ confidence interval

\section{Discussion}

We evaluated whether expression patterns of fascin and BRMS1 correlate with clinicopathological features and patient outcome in breast cancer. A previous study demonstrated that fascin promoted breast cancer invasion via upregulating NF-kB activity, urokinase-type plasminogen activator $(u \mathrm{PA})$, and matrix metalloproteases (MMPs) that are essential to metastasis; in contrast, fascin inhibited the 
nuclear expression of BRMS1 in breast cancers [13]. To assess the importance of the intracellular localization of BRMS1, we separately interpreted the nuclear and cytoplasmic expression of BRMS1. Our results showed that loss of nuclear BRMS1 expression was more frequently observed in fascin ${ }^{+}$tumors (although this was not statistically significant). Cytoplasmic BRMS1 expression and combined expression levels of nuclear and cytoplasmic BRMS1 (defined as the BRMS1 H score) showed a significant inverse correlation with fascin expression, which reflects the fact that intracellular expression of BRMS1 is downregulated by fascin.

The BMRS1 sequence contains nuclear export and import signals that enable its nucleus-cytoplasm shuttling [21]. It is not yet fully understood how the anti-metastatic effects of BRMS1 are influenced by its intracellular localization. Two melanoma studies reported that nuclear expression promoted metastatic capacity, whereas cytoplasmic expression suppressed metastasis and increased DFS [9, 22]. By contrast, a breast cancer study reported that a shifting of BRMS1 from the nucleus to the cytoplasm was associated with aggressive features, such as a high proliferation index and a trend toward worse OS [23]. We found that loss of BRMS1 cytoplasmic expression was associated with shorter DFS, whereas nuclear BRMS1 expression status did not affect patient prognosis.

In this study, fascin positivity was significantly associated with various unfavorable clinicopathological parameters, including higher histological and nuclear grades, hormone receptor negativity, and triple-negative subtypes, as in previous studies $[17,24]$. Such correlations remained when we compared the clinicopathological characteristics of the fascin-/BRMS1 ${ }^{+}$and fascin $^{+}$/BRMS1- tumor subgroups. These results may partially explain why breast cancers with these well-known unfavorable clinicopathological features have a higher possibility of metastasis: They are enriched with metastasis-promoting factors and lack metastasis-suppressing factors.

The only unexpected finding was that axillary lymph node metastasis was observed more frequently in fascin- tumors than in fascin ${ }^{+}$tumors. A few previous studies have found a significant positive correlation between fascin expression and lymph node metastasis $[25,26]$, but other studies have failed, as in this study $[17,24]$ These inconsistent results are probably due to the fact that various factors besides fascin and BRMS1 contribute to lymph node metastasis under complex interrelationships. Another possible reason may be attributed to the nature of the cohort included in our study. After reviewing the patients' clinicopathological data, we found that most of the TNBC patients in our cohort were in the early stage without nodal metastasis. This probably weakened the importance of fascin as a prognostic factor; thus, we performed an additional prognosis analysis confined to patients without nodal metastasis. In the node-negative patient group, fascin was a significant independent predictor of shortened DFS and OS. In addition, the fascin ${ }^{+}$/BRMS1- tumor group showed a shorter OS than the fascin ${ }^{-} /$BRMS1 $^{+}$ tumor group (although this was not statistically significant in multivariate analyses). These results suggest that the fascin positivity and loss of BRMS1 expression contribute to poor clinical outcomes in node-negative breast cancers that generally show a favorable prognosis. In regions where breast cancer screening is widely available, such as in South Korea, node-negative cases account for a high percentage of all breast cancers. About $60 \%$ of Korean breast cancer patients are found to have no lymph node metastasis at the time of initial diagnosis [27, 28]. Adjuvant systemic chemotherapy decreases the risk of distant metastasis and death in about $30 \%$ of patients with node-negative breast cancer, while the remaining 70\% do not achieve additional therapeutic benefit from such treatment [27]. Therefore, it is important to identify biomarkers that can predict and select the patients who are more likely to benefit from adjuvant chemotherapy. To the best of our knowledge, this study is the first to report that positive expression of fascin and/or expressional loss of BRMS1 may be a critical predictor of a poor prognosis in the patients with node-negative breast cancer. For this reason, staining of these two biomarkers is recommended especially in the node-negative patients.

In conclusion, this is the first reported study to examine expression of fascin and BRMS1, a metastatic promoter and suppressor, in a quite large cohort of breast cancer patients. Our results confirm the inverse correlation between expression of the two markers in breast cancer tissues. Fascin alone or combined with BRMS1 has value as a poor prognostic marker, particularly in node-negative breast cancer patients.

\section{Acknowledgments}

This research was supported by a grant of the Korea Health Technology R\&D Project through the Korea Health Industry Development Institute (KHIDI), funded by the Ministry of Health \& Welfare, Republic of Korea (grant number: HI16C1559)

\section{Competing Interests}

The authors have declared that no competing interest exists. 


\section{References}

1. Chaffer CL, Weinberg RA. A perspective on cancer cell metastasis. Science. 2011; 331: 1559-64.

2. Jiang P, Enomoto A, Takahashi M. Cell biology of the movement of breast cancer cells: intracellular signalling and the actin cytoskeleton. Cancer Lett. 2009; 284: 122-30.

3. Yamaguchi H, Condeelis J. Regulation of the actin cytoskeleton in cancer cell migration and invasion. Biochim Biophys Acta. 2007; 1773: 642-52.

4. Yang S, Huang FK, Huang J, Chen S, Jakoncic J, Leo-Macias A, et al. Molecular mechanism of fascin function in filopodial formation. J Biol Chem. 2013; 288: 274-84.

5. Yamashiro S, Yamakita Y, Ono S, Matsumura F. Fascin, an actin-bundling protein, induces membrane protrusions and increases cell motility of epithelial cells. Mol Biol Cell. 1998; 9: 993-1006.

6. Machesky LM, Li A. Fascin: Invasive filopodia promoting metastasis. Commun Integr Biol. 2010; 3: 263-70.

7. Seraj MJ, Samant RS, Verderame MF, Welch DR. Functional evidence for a novel human breast carcinoma metastasis suppressor, BRMS1, encoded at chromosome 11q13. Cancer Res. 2000; 60: 2764-9.

8. Kodura MA, Souchelnytskyi S. Breast carcinoma metastasis suppressor gene 1 (BRMS1): update on its role as the suppressor of cancer metastases. Cancer Metastasis Rev. 2015; 34: 611-8.

9. Slipicevic A, Holm R, Emilsen E, Ree Rosnes AK, Welch DR, Maelandsmo GM, et al. Cytoplasmic BRMS1 expression in malignant melanoma is associated with increased disease-free survival. BMC Cancer. 2012; 12: 73.

10. Yang J, Zhang B, Lin Y, Yang Y, Liu X, Lu F. Breast cancer metastasis suppressor 1 inhibits SDF-1alpha-induced migration of non-small cell lung cancer by decreasing CXCR4 expression. Cancer Lett. 2008; 269: 46-56.

11. Zhang S, Lin QD, Di W. Suppression of human ovarian carcinoma metastasis by the metastasis-suppressor gene, BRMS1. Int J Gynecol Cancer. 2006; 16: 522-31.

12. Zhang Y, Guan J, Sun Y, Chai J, Zou T, Gong W, et al. Effect of BRMS1 on tumorigenicity and metastasis of human rectal cancer. Cell Biochem Biophys. 2014; 70: 505-9.

13. Al-Alwan M, Olabi S, Ghebeh H, Barhoush E, Tulbah A, Al-Tweigeri T, et al. Fascin is a key regulator of breast cancer invasion that acts via the modification of metastasis-associated molecules. PLoS One. 2011; 6: e27339.

14. Lakhani SR, International Agency for Research on Cancer., World Health Organization. WHO classification of tumours of the breast. 4th ed. Lyon: International Agency for Research on Cancer; 2012.

15. Elston CW, Ellis IO. Pathological prognostic factors in breast cancer. I. The value of histological grade in breast cancer: experience from a large study with long-term follow-up. C. W. Elston \& I. O. Ellis. Histopathology 1991; 19; 403-410. Histopathology. 2002; 41: 151-2, discussion 2-3.

16. Edge SB, Compton CC. The American Joint Committee on Cancer: the 7th edition of the AJCC cancer staging manual and the future of TNM. Ann Surg Oncol. 2010; 17: 1471-4.

17. Min KW, Chae SW, Kim DH, Do SI, Kim K, Lee HJ, et al. Fascin expression predicts an aggressive clinical course in patients with advanced breast cancer. Oncol Lett. 2015; 10: 121-30.

18. Park SH, Song JY, Kim YK, Heo JH, Kang H, Kim G, et al. Fascin1 expression in high-grade serous ovarian carcinoma is a prognostic marker and knockdown of fascin1 suppresses the proliferation of ovarian cancer cells. Int J Oncol. 2014; 44: 637-46.

19. Wang CQ, Tang CH, Chang HT, Li XN, Zhao YM, Su CM, et al. Fascin-1 as a novel diagnostic marker of triple-negative breast cancer. Cancer Med. 2016; 5: 1983-8.

20. Hicks DG, Yoder BJ, Short S, Tarr S, Prescott N, Crowe JP, et al. Loss of breast cancer metastasis suppressor 1 protein expression predicts reduced disease-free survival in subsets of breast cancer patients. Clin Cancer Res. 2006; 12 : 6702-8.

21. Rivera J, Megias D, Navas C, Bravo J. Identification of essential sequences for cellular localization in BRMS1 metastasis suppressor. PLoS One. 2009; 4: e6433.

22. Riker AI, Samant RS. Location, location, location: the BRMS1 protein and melanoma progression. BMC Med. 2012; 10: 19.

23. Frolova N, Edmonds MD, Bodenstine TM, Seitz R, Johnson MR, Feng R, et al. A shift from nuclear to cytoplasmic breast cancer metastasis suppressor 1 expression is associated with highly proliferative estrogen receptor-negative breast cancers. Tumour Biol. 2009; 30: 148-59.

24. Yoder BJ, Tso E, Skacel M, Pettay J, Tarr S, Budd T, et al. The expression of fascin, an actin-bundling motility protein, correlates with hormone receptor-negative breast cancer and a more aggressive clinical course. Clin Cancer Res. 2005; 11: 186-92.

25. Youssef NS, Hakim SA. Association of Fascin and matrix metalloproteinase-9 expression with poor prognostic parameters in breast carcinoma of Egyptian women. Diagn Pathol. 2014; 9: 136.

26. Omran OM, Al Sheeha M. Cytoskeletal Focal Adhesion Proteins Fascin-1 and Paxillin Are Predictors of Malignant Progression and Poor Prognosis in Human Breast Cancer. J Environ Pathol Toxicol Oncol. 2015; 34: 201-12.

27. Harbeck N, Thomssen C. A new look at node-negative breast cancer. Oncologist. 2011; 16 Suppl 1: 51-60.
28. Ahn SG, Lee HM, Lee SA, Jeong J, Lee HD. Long-term survival analysis of korean breast cancer patients at a single center: improving outcome over time. Yonsei Med J. 2014; 55: 1187-95. 